\title{
BONE LESIONS IN PIGMENTED VILLONODULAR SYNOVITIS*
}

\author{
P. M. Scott, Cambridge, England \\ From the Orthopaedic Department, Addenbrooke's Hospital, Cambridge
}

Pigmented villonodular synovitis was the term introduced by Jaffe, Lichtenstein and Sutro in 1941 to describe a condition of unknown etiology in which the synovial membrane of a joint becomes diffusely or locally thickened. They described a similar change occurring in the synovium of tendon sheaths and bursae. Macroscopically the synovium is villous or nodular, and varies in colour with the content of iron pigment from yellow and red, to dark brown. Microscopically there is marked proliferation of the surface cells and of the connective tissue throughout the synovial membrane, together with an extraordinary accumulation of intracellular and extracellular haemosiderin and lipoid material, associated with an abundance of reticulo-endothelial cells, including giant cells (Fig. 1).

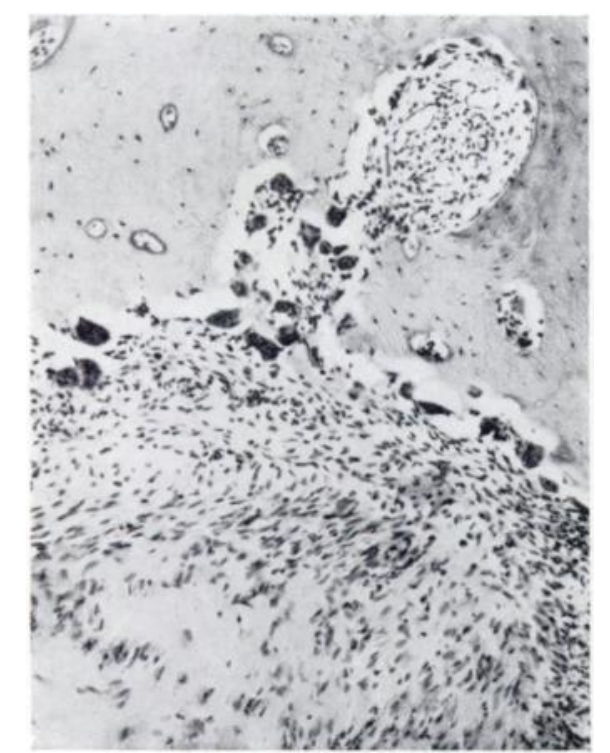

Fig. 1

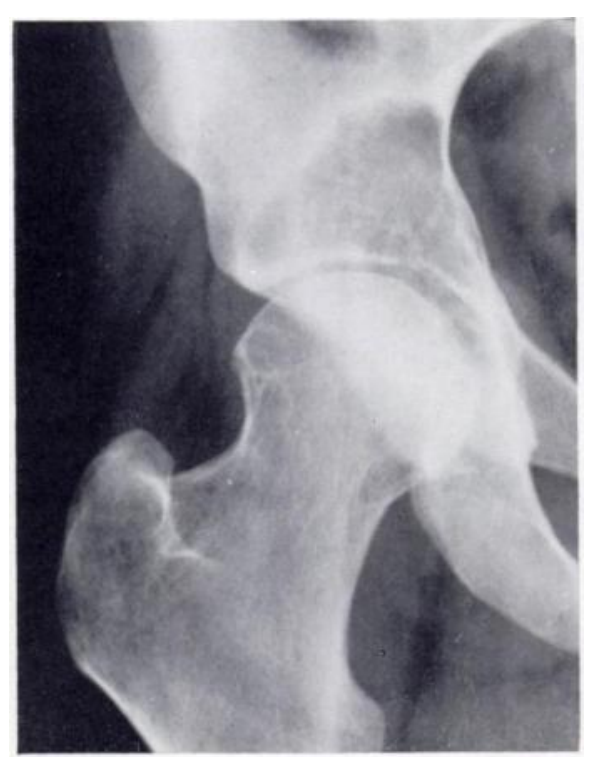

FIG. 2

Figure 1-Photomicrograph showing the synovial membrane affected by pigmented villonodular synovitis with bone involvement. (Haematoxylin and eosin, 85 .) Figure 2 -Radiograph to show the characteristic intraosseous cysts in the femoral neck and acetabulum.

A feature occasionally present is involvement of the bones by villonodular tissue, producing the radiological change (Lewis 1947, Greenfield and Wallace 1950, Breimer and Freiberger 1958) of single or multiple intraosseous cysts, each delineated by a faint line of sclerosis, the surrounding bone being of normal density and the joint space preserved (Fig. 2). These radiological changes, if present, may help to establish the diagnosis before operation.

Twenty-four cases have been reported in the English literature (Mallory, Castleman and Parris 1944; Rogers 1944; Lewis 1947; Clarke 1951; Minear 1951; Carr, Berley and Davis 1954; Ghormley and Romness 1954; Breimer and Freiberger 1958; McMaster 1960; Snook 1963; Chung and Janes 1965). The findings in five more cases, confirmed histologically, form the basis of this paper. Study of the operation and pathology findings has helped to explain the bone lesions and leads to some conclusions about treatment.

\footnotetext{
* Read at the meeting of the British Orthopaedic Association in Cambridge in March 1966.
} 
The bone lesions do not seem to be the result of direct external pressure, as in the case of aneurysms or extraosseous tumours, and they differ from infection or new growths of bone. We believe that invasion of bone takes place through the vascular foramina underlying the diseased synovium. Villonodular tissue grows into these foramina alongside the nutrient vessels, carrying with it its own blood supply. It expands the foramina and, following the path of least resistance along the course of the osseous vessels, may pass deeply into bone where its growth produces intraosseous cysts by a process of pressure atrophy (Fig. 1). No blood supply is got from the intraosseous vessels. For its growth and survival the villonodular tissue in bone is entirely dependent upon its own blood supply.

\section{CASE REPORTS}

Case 1-A woman of twenty-three had chronic swelling and discomfort in her left knee for two years. Arthrotomy revealed pigmented villonodular synovitis of the diffuse type, and partial synovectomy was carried out. Five years later a further synovectomy was done but seven years later the discomfort in her knee had become so bad that arthrodesis was done. At this operation little persisting villonodular change was found in the synovium except in the previously inaccessible intercondylar regions of the joint. Upon removal of a slice of bone from the articular surface of the upper tibia two small nodules of diseased synovial tissue, each less than one centimetre in diameter, were found in the cancellous bone of the tibia below and in front of the insertion of the anterior cruciate ligament (Fig. 3). No evidence of bone involvement was apparent on radiographic examination immediately before operation, presumably because of the small size of these intraosseous lesions. Since arthrodesis the knee has been painless.

Case 2-A woman of thirty-eight had suffered from a swollen and painful knee for eighteen years. At operation the knee was extensively involved with diffuse pigmented villonodular synovitis, and as complete a synovectomy as possible was performed. Radiographs kefore operation showed extensive cystic lesions in the central area of the upper tibia and of the femur (Fig. 4) and it seemed probable that the route of entry of villonodular tissue was through the vascular foramina in the intercondylar areas of the tibia and femur. The intraosseous cysts were explored and packed with cancellous autogenous bone grafts at a later operation. Radiotherapy was given after operation. Radiographs some months later showed regression of the bone lesions (Fig. 5).

Case 3-A man of thirty-six had pain in the right hip for five years. Radiographic examination at this time (Fig. 6) showed numerous cystic defects in the

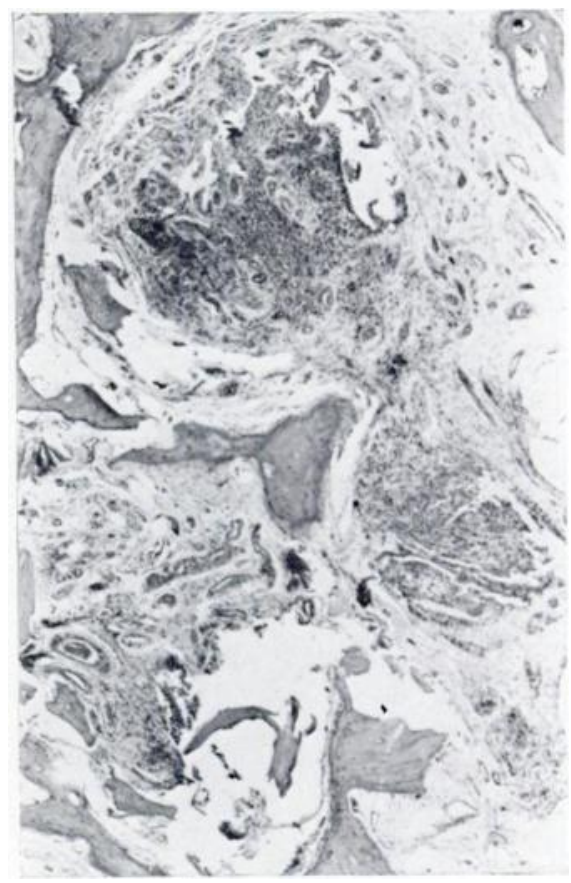

FIG. 3

Case 1-Photomicrograph of villonodular synovial tissue lying within the cancellous bone of the upper tibia. (Haematoxylin and eosin, $\times 35$.) femoral neck and head, together with a large cyst above and behind the acetabulum. The joint space, however, remained well preserved and the surrounding bone was of normal appearance. Later the same year the hip was explored through a Smith-Petersen incision, and when the distended capsule was opened the whole synovium was found to be replaced by masses of pigmented villonodular synovial tissue. Synovectomy was performed, necessarily incomplete, as the hip was not dislocated at operation. Where the villonodular tissue had invaded the femoral neck the processes were running towards the femoral head, and pulling on the bases of these tissue processes often resulted in their complete extraction from the bone, revealing smooth-walled avascular cavities. The ilium was explored and revealed a large cavity full of villonodular tissue which was in part necrotic but otherwise similar to that within the hip. This cavity opened into the acetabular fossa, leaving no doubt that invasion of the ilium had occurred along this route.

Radiotherapy was given afterwards, but some months later a pathological fracture developed in the femoral neck. Fortunately union occurred leaving the patient with a reasonably comfortable but rather stiff hip. It is interesting that the cyst in the ilium, although not grafted, became largely obliterated over a period of four years (Fig. 7).

VOL. $50 \mathrm{~B}$, NO. 2, MAY 1968 


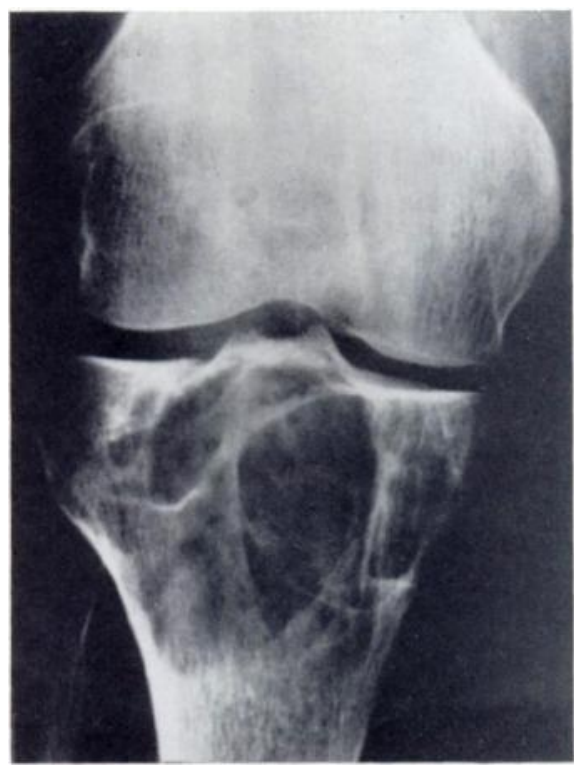

Fig. 4

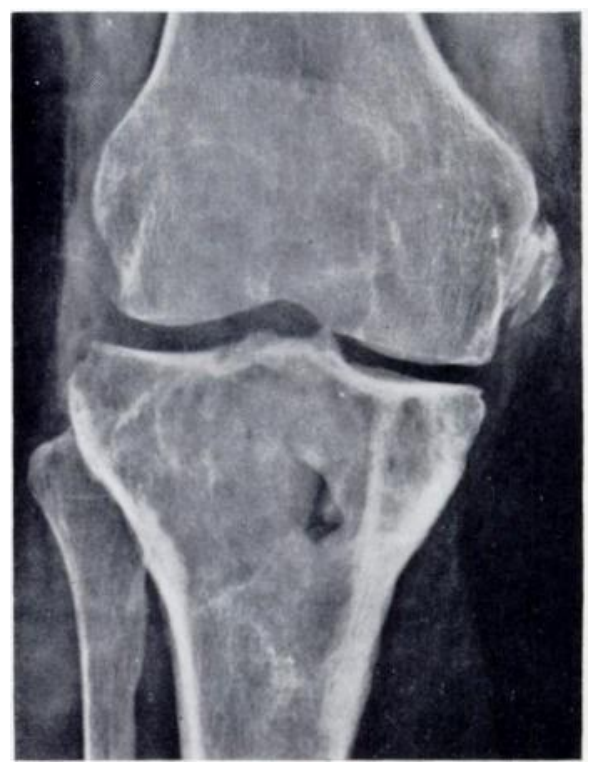

Fig. 5

Case 2. Figure 4-Radiograph of the knee showing extensive cystic lesions in the upper tibia and several small lesions in the intercondylar region of the lower femur. Figure 5-Radiographs of the knee after packing with autogenous bone grafts and radiotherapy.

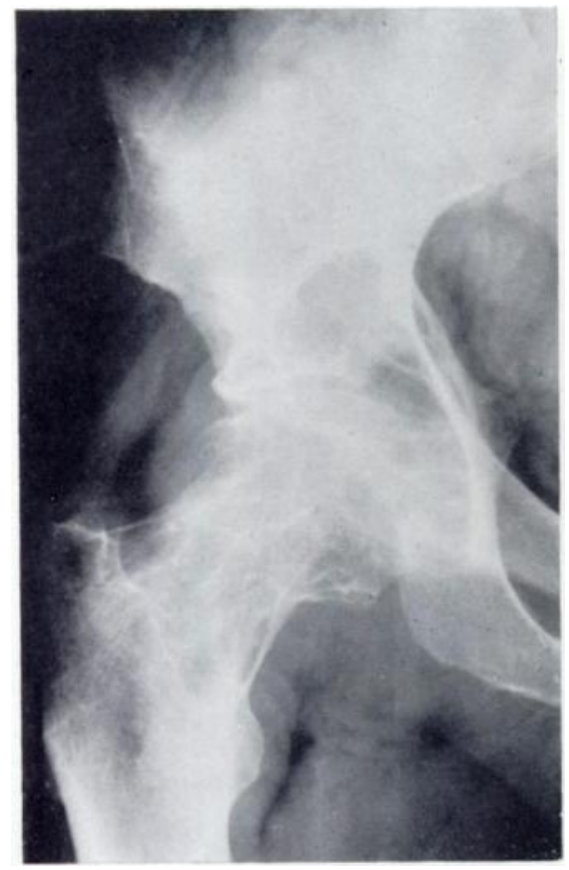

FIG. 6

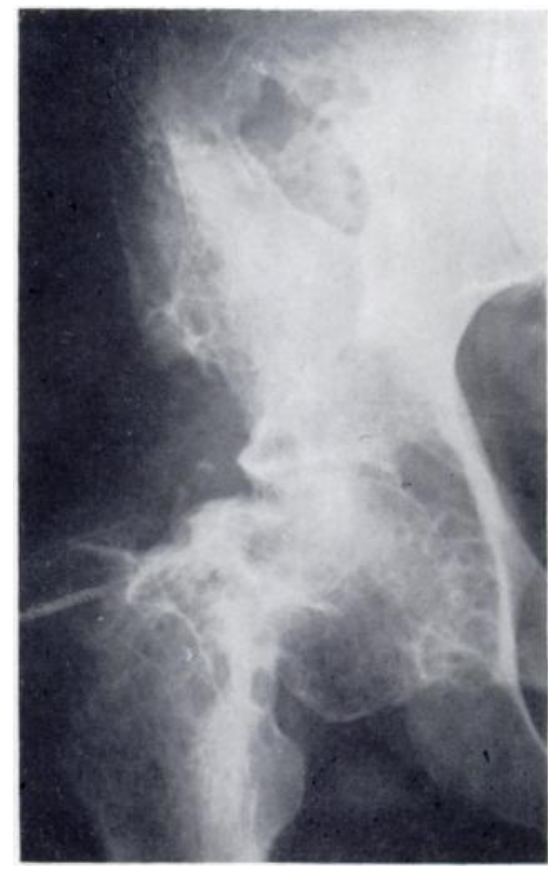

Fig. 7

Case 3. Figure 6-Radiograph of the hip showing numerous intraosseous cystic lesions of pigmented villonodular synovitis in the femoral neck and head and around the acetabulum. Figure 7-Radiograph showing the appearance of the hip four years later. The pathological fracture of the femoral neck has united and the cyst in the ilium has largely disappeared. 
Case 4-A woman of twenty-eight had pain in the right hip after activity, getting worse for five months. Examination showed no abnormality and the radiographs were diagnostic. The hip was explored through a posterior incision, and the operation findings were similar to those in the previous case. In addition, there was involvement of the bursa deep to the quadratus femoris muscle (Carr et al. 1954, McMaster 1960). In order to facilitate the complete removal of abnormal synovium the femoral head was dislocated, revealing extensive involvement of the acetabulum (Figs. 8 and 9). Exploration of the cyst in the ilium revealed masses of partly necrotic villonodular tissue communicating with the

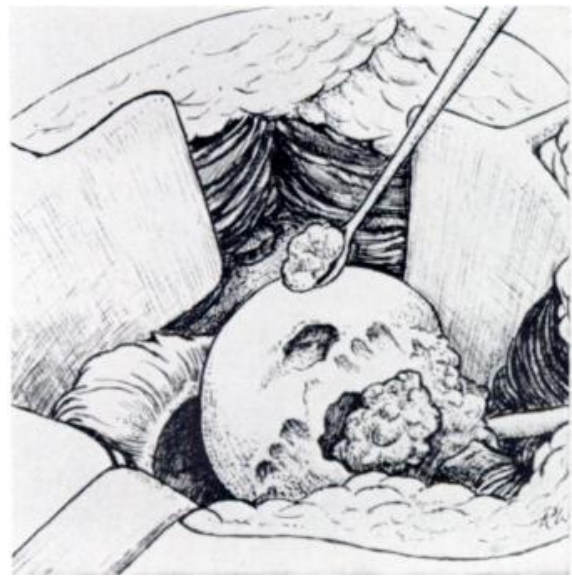

Fig. 8

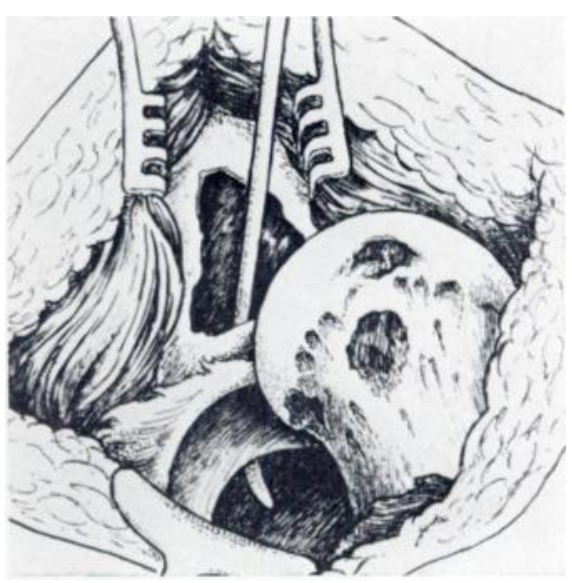

Fig. 9

Case 4. Figure 8-The removal of villonodular tissue from the bone cavities in the femoral neck. Figure 9-Showing the dislocated femoral head, the openings into the bone cavities in the femoral neck and the obvious communication between the acetabulum and the large iliac cyst.

hip through the acetabulum. This cavity was packed with autogenous cancellous bone grafts. Radiotherapy was not given after operation. At follow-up two years later the patient remained well and symptom-free; the hip was functionally normal and some regression of the intraosseous bone lesions had occurred.

Case 5-A young woman with pigmented villonodular synovitis of the elbow (histologically confirmed) with extensive cystic bone involvement of the lower humerus and upper ulna showed all the radiological features of this condition. This case was considered unsuitable for radical surgery due to the extensive bone involvement in the vicinity of the elbow. The condition in this case has been well controlled by radiotherapy for some years.

\section{DISCUSSION}

It is apparent that bone involvement in pigmented villonodular synovitis occurs by the growth of villonodular tissue through the vascular foramina and that the blood supply of the intraosseous synovium is conveyed along vascular stalks.

Radiologically the cysts always appear to originate in areas of bone within the joint capsule where the vascular foramina are both large and numerous. In the hip these foramina are found on the femoral neck, particularly close to the articular margin, and also in the acetabular fossa and in the fovea capitis. In the knee the intercondylar area of the tibia and femur are the usual sites. At operation small fingers of villonodular tissue can be seen entering the vascular foramina which are recognisable as such by their site, shape and direction even though pressure erosion may have begun to enlarge them. Traction on the base of such a process entering bone may result in its removal intact from the bone. There is no attachment of villonodular tissue to the walls of the cavity nor are there vascular connections with the intraosseous blood vessels. In cases with large cysts in bone, for example in the ilium above the acetabulum, the point of entry of villonodular synovium could be demonstrated in the acetabulum, and in places the synovium within these cysts was necrotic, suggesting that it had outgrown its blood supply. Increased intracapsular pressure was always found at operation, 
due to the great mass of villonodular tissue and tense effusion. Possibly aggravated by the pumping action of certain joint movements, it may be a factor in encouraging bone involvement.

The strong unyielding capsule of the hip, as compared with the more lax and accommodating capsule of the knee, must result in a relatively greater intracapsular pressure and may explain the fact that in the previously recorded cases of this condition with bone involvement fourteen were in the hip but only three in the knee, whereas villonodular synovitis without bone involvement is ten or fifteen times more common in the knee than in the hip (Jaffe 1960).

We are not in agreement with the explanation of the formation of bone lesions as propounded by McMaster (1960), who stated that extension of pigmented villonodular synovitis appeared to be through articular cartilage and cortex at the chondro-osseous junction; nor can we accept the suggestion of Chung and Janes (1965) who believed that synovial overgrowth leads to generalised increase in joint pressure which in turn leads to small areas of osteoporosis, this being followed by cystic degeneration in bone and finally by fracture of the cyst walls and invasion by exuberant synovial membrane.

There is evidence that radiotherapy controls the intraosseous spread of villonodular synovitis (Friedman and Schwartz 1957), and this may prove to be the best form of treatment for spread into areas not easily accessible to surgery (as in Cases 2, 3 and 5). In one of the hips (Case 3), however, this may have been a contributory factor to late pathological fracture of the femoral neck.

It is interesting that in the other hip case (Case 4) the femoral head was dislocated and the ligamentum teres excised to allow radical synovectomy, but no avascular changes in the femoral head followed. This suggests that the circulation of blood to the femoral head may have become gradually adapted to survive on the intramedullary vessels ascending within the femoral neck, rather than on vessels entering the vascular foramina around the femoral neck, which may have been gradually obliterated by the pressure of the invading villonodular synovial tissue.

From our small experience of these cases of pigmented villonodular synovitis with bone involvement, we agree with McMaster (1960) that radical synovectomy should remain the main basis of treatment, with particular attention to removing the stalks of all synovial processes entering bone. Exploration of the cystic areas in bone, although desirable, may prove to be unnecessary if the stalks of diseased tissue entering them are divided.

Radiotherapy is of undoubted value in cases in which synovectomy is inadequate or where operative treatment is technically difficult.

\section{SUMMARY}

1. Five cases of pigmented villonodular synovitis with associated lesions within bone are recorded, two in the hip, two in the knee and one in the elbow.

2. The mode of formation of these intraosseous lesions is explained and methods of treatment are discussed.

I wish to thank Mr R. W. Butler and Mr T. J. Fairbank for their help, encouragement and criticism in the preparation of this paper and for permission to study Cases 1 and 3; also to $\mathrm{Mr} \mathrm{J}$. Wishart for Cases 4 and 5 , and Mr G. Storrs for Case 2. I am grateful to Dr J. H. Rack and Mr S. Patman of the Pathology Department, University of Cambridge, for help in the preparation of the photomicrographs, and to the staff of the Photographic Department of Addenbrooke's Hospital, Cambridge, for the reproduction of radiographs. I am indebted to

Mrs E. Thoday for her assistance with the preparation and typing of the script. Case 1 has been recorded before the development of bone lesions by Mr G. R. Fisk (1952): Annals of the Royal College of Surgeons of England, 11, 157.

\section{REFERENCES}

Breimer, C. W., and Freiberger, R. H. (1958): Bone Lesions Associated with Villonodular Synovitis. American Journal of Roentgenology, Radium Therapy and Nuclear Medicine, 79, 618.

Carr, C. R., Berley, F. V., and Davis, W. C. (1954): Pigmented Villonodular Synovitis of the Hip Joint. A Case Report. Journal of Bone and Joint Surgery, 36-A, 1007. 
Chung, S. M. K., and Janes, J. M. (1965): Diffuse Pigmented Villonodular Synovitis of the Hip Joint. Journal of Bone and Joint Surgery, 47-A, 293.

Clark, W. S. (1951): Case Records of the Massachusetts General Hospital. Case 37292. New England Journal of Medicine, 245, 113.

Fisk, G. R. (1952): Hyperplasia and Metaplasia in Synovial Membrane. Annals of the Royal College of Surgeons of England, 11, 157.

Friedman, M., and Schwartz, E. E. (1957): Irradiation Therapy of Pigmented Villonodular Synovitis. Bulletin of the Hospital for Joint Diseases, 18, 19.

Ghormley, R. K., and Romness, J. O. (1954): Pigmented Villonodular Synovitis (Xanthomatosis) of the Hip Joint. Proceedings of the Staff Meetings of the Mayo Clinic, 29, 171.

Greenfield, M. M., and Wallace, K. M. (1950): Pigmented Villonodular Synovitis. Radiology, 54, 350.

JAfFe, H. L. (1960): Discussion of Pigmented Villonodular Synovitis with Invasion of Bone. Report of Six Cases by Paul E. McMaster. Journal of Bone and Joint Surgery, 42-A, 1183.

Jaffe, H. L., Lichtenstein, L., and Sutro, G. J. (1941): Pigmented Villonodular Synovitis, Bursitis and Tenosynovitis. Archives of Pathology, 31, 731.

Lewis, R. W. (1947): Roentgen Diagnosis of Pigmented Villonodular Synovitis and Synovial Sarcoma of the Knee Joint. Radiology, 49, 26.

MCMaster, P. E. (1960): Pigmented Villonodular Synovitis with Invasion of Bone. Journal of Bone and Joint Surgery, 42-A, 1170.

Mallory, T. B., Castleman, B., and Parris, E. E. (1944): Case Records of the Massachusetts General Hospital. Case 30131. New England Journal of Medicine, 230, 409.

Minear, W. L. (1951): Xanthomatous Joint Tumors. Journal of Bone and Joint Surgery, 33-A, 451.

Rogers, W. A. (1944): Case Records of the Massachusetts General Hospital. Case 30131. New England Journal of Medicine, 230, 410.

SNOOK, G. A. (1963): Pigmented Villonodular Synovitis with Bony Invasion; A Report of Two Cases. Journal of the American Medical Association, 184, 424.

YounG, J. M., and HudaceK, A. G. (1954): Experimental Production of Pigmented Villonodular Synovitis in Dogs. American Journal of Pathology, 30, 799. 